\title{
Relationship between Estradiol and Antioxidant Enzymes Activity of Ischemic Stroke
}

\author{
Nasrin Sheikh, ${ }^{1}$ Heidar Tavilani, ${ }^{1}$ Aliakbar Rezaie, ${ }^{2}$ Asad Vaisi-raygani, ${ }^{3}$ and Saeedeh Salimi ${ }^{4}$ \\ ${ }^{1}$ Department of Biochemistry \& Nutrition, School of Medicine, Hamadan University of Medical Sciences, \\ 65178/518 Hamadan, Iran \\ ${ }^{2}$ Department of Neurology, Hamadan University of Medical Sciences, 65178/518 Hamadan, Iran \\ ${ }^{3}$ Department of Clinical Biochemistry, Faculty of Medicine, Kermanshah University of Medical Science, \\ 6714869914 Kermanshah, Iran \\ ${ }^{4}$ Department of Biochemistry, Faculty of Medicine, Zahedan University of Medical Science, 4318198167 Zahedan, Iran
}

Correspondence should be addressed to Heidar Tavilani, tavilani@gmail.com

Received 26 March 2009; Revised 5 July 2009; Accepted 24 July 2009

Recommended by Xudong Huang

Some evidence suggests the neuroprotection of estrogen provided by the antioxidant activity of this compound. The main objective of this study was to determine the level of estradiol and its correlation with the activity of antioxidant enzymes, total antioxidant status and ferritin from ischemic stroke subjects. The study population consisted of 30 patients with acute ischemic stroke and 30 controls. There was no significant difference between estradiol in stroke and control group. The activity of superoxide dismutase and level of ferritin was higher in stroke compared with control group $(P<.05, P<.001$, resp. $)$. There was no significant correlation between estradiol and glutathione peroxidase, glutathione reductase, catalase, total antioxidant status, and ferritin in stroke and control groups. We observed inverse correlation between estradiol with superoxide dismutase in males of stroke patients $(r=-0.54, P=.029)$. Our results supported that endogenous estradiol of elderly men and women of stroke or control group has no antioxidant activity.

Copyright () 2009 Nasrin Sheikh et al. This is an open access article distributed under the Creative Commons Attribution License, which permits unrestricted use, distribution, and reproduction in any medium, provided the original work is properly cited.

\section{Introduction}

Stroke is the third most common cause of death, particularly in the elderly. Ischemic stroke accounts for about $75 \%$ of all cases while hemorrhagic stroke is responsible for almost $15 \%$ of all strokes [1]. Data on the link between endogenous estrogen and ischemic stroke in elderly men and women is limited and controversial [2]. Before the menopause, women are relatively protected from ischemic stroke compared with men. This protection is most attributed to the action of estrogen $[3,4]$ but recent evidence has raised the possibility that sex-dependent differences in vascular oxidative stress may play a role [5]. In postmenopausal women and elderly men, the role of endogenous estrogen is not clear. Some studies have found that hyperestrogenemia is related to an elevated risk of coronary heart disease, although other studies have not [6]. Abbott et al. found elderly men with high level of serum estradiol have twofold excess risk of stroke compared with men whose estradiol levels were lower [6]. Relationship between endogenous estradiol and antioxidant status of elderly men and women from stroke is less known. Some evidences suggest the neuroprotective of estrogen or estrogen derivatives provided by the antioxidant activity of this compound [7], but some studies are observed the antioxidant activity of estrogen, only with supraphysiologic dose of estrogen [8].

Since oxidative stress is important mechanism involved in ischemic stroke, the activity of antioxidant enzymes may be an essential factor providing protection from neurological damage $[1,9,10]$. Ferretti et al. found the higher level of lipid hydroperoxides in plasma from stroke patients compared with control group [11]. The question whether endogenous estradiol can affect the activity of antioxidant enzyme or level of ferritin in the ischemic stroke has not been systematically addressed yet, although the activity of antioxidant enzyme and level of ferritin from stroke patients have been reported 
$[9,10,12,13]$. To the best of our knowledge, no information is available about correlation between endogenous estradiol and the activity of antioxidant enzymes from ischemic stroke patients. The main objective of this study was to determine the level of estradiol and its correlation with the activity of antioxidant enzymes and ferritin from ischemic stroke subjects.

\section{Materials and Methods}

Thirty patients older than 61 years with acute ischemic stroke admitted within 12 hours from the onset of symptoms to the emergency room of the Farshchian University Hospital, Hamadan, Iran, and were consecutively enrolled. On admission, demographic characteristics, a detailed history, and clinical information for stroke risk factors were recorded. Laboratory tests (blood cell count, biochemical studies including lipid profiles and serum electrolytes, urine analysis), chest radiography, and electrocardiography were performed in all patients. All patients were investigated to clarify etiologic factors for stroke. Computed tomography (CT) of the brain was performed in all patients. Subjects with hemorrhagic stroke, other neurological disease, patients with body temperature higher than $37.5^{\circ} \mathrm{C}$, inflammatory process, diabetes mellitus, liver disease, and renal impairment, or taking iron or antioxidant vitamins or hormone replacement therapy during the months preceding the enrollment, were excluded from the study. The severity of the neurological deficit was determind by Canadian Neurological Scale [14]. Heparinized venous blood samples were taken and plasma was separated and stored at $-70^{\circ} \mathrm{C}$. Results from the patient group were compared with those obtained from 30 healthy subjects of comparable age and gender. None of the control subjects was undergoing pharmacological treatments. All subjects gave informed consent to participate in the study, according to the criteria of the Ethical Committee of Hamadan University of Medical Sciences.

2.1. Estradiol Assay. Plasma 17- $\beta$ estradiol was measured by a quantitative competitive immunoassay kit (DRG Instruments, Germany).

2.2. Glutathione Peroxidase Assay. Whole blood glutathione peroxidase was measured by a kit from Ransel (Randox Laboratories, UK). Glutathione peroxidase catalyses the oxidation of glutathione by cumene hydroperoxide. In the presence of glutathione reductase and NADPH, the oxidised glutathione is immediately converted to the reduced form with a concomitant oxidation of NADPH to $\mathrm{NADP}^{+}$. The decrease in absorbance at $340 \mathrm{~nm}$ is measured. The GPX activity is expressed as $\mathrm{U} / \mathrm{L}$.

2.3. Superoxide Dismutase Assay. Whole blood superoxide dismutase activity was measured by a Kit from Ransod (Randox Laboratories, UK), employing xanthine and xanthine oxidase to generate superoxide radicals which react with 2-(4-iodophenyl)-3-(4-nitrophenol)-5phenyltetrazolium chloride (INT) to form a red formazan dye. One unit SOD was the amount that causes a $50 \%$ inhibition in the rate of reduction of INT.
2.4. Total Antioxidant Status Assay. Plasma total antioxidant status was measured by a Kit from Randox Company.

ABTS (2,2-Azino-di-[3-ethylbenzthiazoline sulphonate]) is incubated with a peroxidase (metmyoglobin) and $\mathrm{H}_{2} \mathrm{O}_{2}$ to produce the radical cation $\mathrm{ABTS}^{+}$. Antioxidants in the plasma cause suppression of cation $\mathrm{ABTS}^{+}$to a degree that is proportional to their concentration.

2.5. Glutathione Reductase Assay. Glutathione reductase activity of the erythrocytes was measured by a Kit from Randox Company. Glutathione reductase catalyzes the reduction of glutathione in the presence of NADPH, which is oxidized to $\mathrm{NADP}^{+}$. The decrease in absorbance at $340 \mathrm{~nm}$ is measured.

2.6. Catalase Assay. Catalase activity of the erythrocytes was measured according to Abei (15) by monitoring the initial rate of disappearance of hydrogen peroxide (initial concentration $10 \mathrm{mM}$ ) at $240 \mathrm{~nm}$. The catalase activity is expressed as k/g Hb.

2.7. Ferritin Assay. Ferritin levels of the plasma were measured by a kit (Pyesthaste Company, Iran) using immunoassay technique.

\section{Statistical Analysis}

Results are expressed as means \pm SD. Kolmogorov-Smirnov goodness of test was used for normality distribution of estradiol, total antioxidant status, superoxide dismutase, glutathione peroxidase, glutathione reductase, catalase, and ferritin. For comparing estradiol, total antioxidant status, superoxide dismutase, glutathione peroxidase, glutathione reductase, catalase, and ferritin between stroke and control groups, the Student's $t$ test was used. In addition, correlation analyses were performed by means of the Pearson test. A value of $P<.05$ was considered significant.

\section{Results}

The study population consisted of 60 subjects: 30 patients with acute ischemic stroke admitted within 12 hours from onset ( 15 men and 15 women, aged $70.6 \pm 8.3$ and $70.4 \pm 10.8$ years, resp.) and 30 controls (15 men and 15 women, aged $68.5 \pm 8.4$ and $66.2 \pm 7.6$ years, resp.). The mean CNS score in stroke group was $6.3 \pm 1.5$.

4.1. Results of Total Subjects from Stroke and Control Group. The mean values of the estradiol, glutathione peroxidase, glutathione reductase, catalase, superoxide dismutase, total antioxidant status, and ferritin in the stroke and control groups are shown in Table 1. There was no significant difference between estradiol in stroke and control group. The activity of superoxide dismutase and level of ferritin was higher in stroke compared with control group $(P<.05$, $P<.001$, resp.). The activity of glutathione peroxidase was lower in stroke compared with control group $(P<.05)$. 
TABLE 1: The mean \pm SD values of the estradiol, ferritin and total antioxidant status, superoxide dismutase and glutathione peroxidase, glutathione reductase and catalase of the stroke and control group.

\begin{tabular}{lcc}
\hline Parameters & Stroke $(n=30)$ & Control $(n=30)$ \\
\hline${\text { Estradiol }(\mathrm{pg} / \mathrm{mL})^{\dagger}}^{\text {Superoxide dismutase }}{ }^{\ddagger}(\mathrm{U} / \mathrm{mL})$ & $63.8 \pm 19.8$ & $66.5 \pm 9.6$ \\
Glutathione peroxidase $^{\ddagger}(\mathrm{U} / \mathrm{L})$ & $174.53 \pm 84.81^{*}$ & $123.94 \pm 85.09$ \\
Glutathione Reductase $^{\#}(\mathrm{U} / \mathrm{g} \mathrm{Hb})$ & $3993.40 \pm 2696.19^{*}$ & $5775.27 \pm 3348.77$ \\
${\text { Catalase }(\mathrm{k} / \mathrm{g} \mathrm{Hb})^{\#}}^{\text {Total antioxidant status }(\mathrm{mmol} / \mathrm{L})^{\dagger}}$ & $2.78 \pm 1.87$ & $3.03 \pm 1.93$ \\
${\text { Ferritin }(\mathrm{ng} / \mathrm{mL})^{\dagger}}$ & $492.97 \pm 115.39$ & $456.11 \pm 103.96$ \\
\hline
\end{tabular}

${ }^{\dagger}$ In plsama, ${ }^{\ddagger}$ in whole blood, ${ }^{\#}$ in erythrocyte.

${ }^{*} P<.05,{ }^{*} P<<.001$.

TABLE 2: The mean \pm SD values of the estradiol, ferritin and total antioxidant status, superoxide dismutase and glutathione peroxidase, glutathione reductase and catalase of the males from stroke and control groups.

\begin{tabular}{lcc}
\hline Parameter & Stroke $(n=15)$ & Control $(n=15)$ \\
\hline Estradiol $^{\dagger}(\mathrm{pg} / \mathrm{mL})$ & $70 \pm 19.6$ & $64.5 \pm 6.9$ \\
Superoxide dismutase $^{\ddagger}(\mathrm{U} / \mathrm{mL})$ & $176.8 \pm 89.6^{*}$ & $112.5 \pm 62.6$ \\
Glutathione peroxidase $^{\ddagger}(\mathrm{U} / \mathrm{L})$ & $4107.9 \pm 3132.8$ & $5791 \pm 3913$ \\
Glutathione reductase $^{\#}(\mathrm{U} / \mathrm{g} \mathrm{Hb})$ & $2.51 \pm 1.98$ & $3.7 \pm 1.92$ \\
Catalase $^{\#}(\mathrm{k} / \mathrm{g} \mathrm{Hb})$ & $504.8 \pm 122.7$ & $406.7 \pm 123.4$ \\
Total antioxidant status $^{\dagger}(\mathrm{mmol} / \mathrm{L})$ & $1.05 \pm 0.26$ & $1.2 \pm 0.34$ \\
Ferritin $^{\dagger}(\mathrm{ng} / \mathrm{mL})$ & $111.9 \pm 60.5^{*}$ & $73 \pm 35.3$ \\
\hline
\end{tabular}

${ }^{\dagger}$ In plsama, ${ }^{\ddagger}$ in whole blood, ${ }^{*}$ in erythrocyte.

${ }^{*} P<.05$.

4.2. Results of Males and Females from Stroke and Control Group. The mean \pm SD values of the estradiol, ferritin, and total antioxidant status, superoxide dismutase and glutathione peroxidase, glutathione reductase, and catalase of the males and females from stroke and control groups are shown in Tables 2 and 3.

The correlation between estradiol and glutathione peroxidase, glutathione reductase, catalase, total antioxidant status and ferritin in males and females from stroke and control groups was not significant. There was inverse correlation between estradiol with superoxide dismutase in males of stroke patients $(r=-0.54, P=.029)$. In addition, we found inverse correlation between estradiol with superoxide dismutase in females of stroke patients, but this difference was not significant $(r=-0.48, P=.13)$.

\section{Discussion}

In the present study, we have determined levels of estradiol, ferritin, total antioxidant status, and activity of antioxidant enzyme in ischemic stroke patients. Because of difficulties in determination of antioxidant enzymes in human cerebral tissue, the measurements were exclusively carried out in blood samples. Data on the relationship of endogenous estradiol and cerebrovascular disease in elderly men and women are limited [2]. In our study, there was no difference between estradiol in stroke and control group. This finding suggests that the level of endogenous estradiol cannot prevent of occurrence of ischemic stroke. Our results are consistent with Abbott et al. who reported high levels of serum estradiol that cannot protect elderly men against stroke [6].

In the present study, ischemic stroke was associated with increased plasma activity of superoxide dismutase during the 12 hours after stroke. Because, after cerebral ischemia and particularly reperfusion, free-radical production is dramatically elevated [15], thus increased superoxide dismutase activity in stroke group may be a compensatory response to oxidative stress in stroke. The result is in agreement with Kocaturk et al. who have reported increased levels of superoxide dismutase in red blood cells after cerebrovascular accident [16].

In the present study, we did not observe positive correlation between estradiol and glutathione peroxidase, glutathione reductase, catalase, total antioxidant status, or ferritin in stroke or control group. In contrast, we found the negative correlation between estradiol and superoxide dismutase activity in males from stroke group. These data suggest a possible effect of estradiol reduction on increase of supreoxide anion and induce oxidative stress in these patients. We suggested that increased of superoxide anion could induce increased superoxide dismutase activity in males of stroke patients which may be a compensatory response to oxidative stress in these patients. However, we observed inverse correlation between estradiol and superoxide dismutase activity in females of stroke group, but the difference was not significant. The result is in agreement with Bertrand et al. who have reported increased 
TABLE 3: The mean \pm SD values of the estradiol, ferritin and total antioxidant status, superoxide dismutase and glutathione peroxidase, glutathione reductase and catalase of the females from stroke and control groups.

\begin{tabular}{lcc}
\hline Parameter & Stroke $(n=15)$ & Control $(n=15)$ \\
\hline Estradiol $^{\dagger}(\mathrm{pg} / \mathrm{mL})$ & $55.4 \pm 17.6$ & $69 \pm 12$ \\
Superoxide dismutase $^{\ddagger}(\mathrm{U} / \mathrm{mL})$ & $171.4 \pm 81.5$ & $137 \pm 106.5$ \\
Glutathione peroxidase $^{\ddagger}(\mathrm{U} / \mathrm{L})$ & $3861.2 \pm 2206.9$ & $5758 \pm 2759$ \\
Glutathione reductase $^{\#}(\mathrm{U} / \mathrm{g} \mathrm{Hb})$ & $3.15 \pm 1.72$ & $2.2 \pm 1.65$ \\
Catalase $^{\#}(\mathrm{k} / \mathrm{g} \mathrm{Hb})$ & $476.9 \pm 106.8$ & $451.1 \pm 82.3$ \\
Total antioxidant status $^{\dagger}(\mathrm{mmol} / \mathrm{L})$ & $1.06 \pm 0.25$ & $1.02 \pm 0.31$ \\
Ferritin $^{\dagger}(\mathrm{ng} / \mathrm{mL})$ & $87.6 \pm 50.7$ & $58.4 \pm 23.6$ \\
\hline
\end{tabular}

${ }^{\dagger}$ In plsama, ${ }^{\ddagger}$ In whole blood, ${ }^{\#}$ In erythrocyte.

level of serum total antioxidant status in postmenopausal women after estrogen replacement therapy [17]. In the other hand, our data suggests that endogenous estradiol do not have antioxidant activity in stroke and control group with age greater than 61 years. Some reports attributed an antioxidant activity to estradiol but the antioxidant activity is observed only with supraphysiologic concentration of this hormone $[8,18,19]$. In the current report, evidence suggests that neuroprotective effects of endogenous estradiol against stroke are not related to antioxidant effect of this compound. It is not clear whether estrogen therapy has potential effect on oxidative stress in stroke patients. It seems that more studies are needed to make clear the role of estrogen therapy on antioxidant status of ischemic stroke patients.

In this study, we observed higher level of ferritin in stroke compared with control group. Recent clinical studies have been proposed that high plasma levels of ferritin might be associated with increased risk of ischemic stroke [12, 13]. Ferritin might induce oxidative stress via being a donor of free iron that is a source of hydroxyl radicals.

There are some limitations in the present study. First, small number of stroke and control individuals. Second, in this study, we did not determine correlations between estradiol and reactive oxygen species or some metabolite of lipid and DNA peroxidation such as malondialdehyde and 8oxoguanine in ischemic stroke group. Thus, further studies are needed to clarify the role of endogenous estradiol on antioxidant status of ischemic stroke patients.

\section{References}

[1] M. A. de Leciñana, J. A. Egido, C. Fernández, et al., "Risk of ischemic stroke and lifetime estrogen exposure," Neurology, vol. 68, no. 1, pp. 33-38, 2007.

[2] M. Muller, Y. T. van der Schouw, J. H. H. Thijssen, and D. E. Grobbee, "Endogenous sex hormones and cardiovascular disease in men," Journal of Clinical Endocrinology and Metabolism, vol. 88, no. 11, pp. 5076-5086, 2003.

[3] P. Jousilahti, E. Vartiainen, J. Tuomilehto, and P. Puska, "Sex, age, cardiovascular risk factors, and coronary heart disease: a prospective follow-up study of 14786 middle-aged men and women in Finland," Circulation, vol. 99, no. 9, pp. 1165-1172, 1999.

[4] D. W. Brann, K. Dhandapani, C. Wakade, V. B. Mahesh, and M. M. Khan, "Neurotrophic and neuroprotective actions of estrogen: basic mechanisms and clinical implications," Steroids, vol. 72, no. 5, pp. 381-405, 2007.

[5] A. A. Miller, T. M. De Silva, K. A. Jackman, and C. G. Sobey, "Effect of gender and sex hormones on vascular oxidative stress," Clinical and Experimental Pharmacology and Physiology, vol. 34, no. 10, pp. 1037-1043, 2007.

[6] R. D. Abbott, L. J. Launer, B. L. Rodriguez, et al., "Serum estradiol and risk of stroke in elderly men," Neurology, vol. 68, no. 8, pp. 563-568, 2007.

[7] K. Prokai-Tatrai, P. Perjesi, N. M. Rivera-Portalatin, J. W. Simpkins, and L. Prokai, "Mechanistic investigations on the antioxidant action of a neuroprotective estrogen derivative," Steroids, vol. 73, no. 3, pp. 280-288, 2008.

[8] M. Alonso de Lecinana and J. A. Egido, "Estrogens as neuroprotectants against ischemic stroke," Cerebrovascular Diseases, vol. 21, supplement 2, pp. 48-53, 2006.

[9] C. Zimmermann, K. Winnefeld, S. Streck, M. Roskos, and R. L. Haberl, "Antioxidant status in acute stroke patients and patients at stroke risk," European Neurology, vol. 51, no. 3, pp. 157-161, 2004.

[10] A. Cherubini, M. C. Polidori, M. Bregnocchi, et al., "Antioxidant profile and early outcome in stroke patients," Stroke, vol. 31, pp. 2295-2300, 2000.

[11] G. Ferretti, T. Bacchetti, S. Masciangelo, et al., "Lipid peroxidation in stroke patients," Clinical Chemistry and Laboratory Medicine, vol. 46, no. 1, pp. 113-117, 2008.

[12] T. Carbonell and R. Rama, "Iron, oxidative stress and early neurological deterioration in ischemic stroke," Current Medicinal Chemistry, vol. 14, no. 8, pp. 857-874, 2007.

[13] D. L. van der A, D. E. Grobbee, M. Roest, J. J. M. Marx, H. A. Voorbij, and Y. T. van der Schouw, "Serum ferritin is a risk factor for stroke in postmenopausal women," Stroke, vol. 36, no. 8, pp. 1637-1641, 2005.

[14] R. Cote, R. N. Battista, C. Wolfson, J. Boucher, J. Adam, and V. Hachinski, "The Canadian Neurological Scale: validation and reliability assessment," Neurology, vol. 39, no. 5, pp. 638-643, 1989.

[15] I. Margaill, M. Plotkine, and D. Lerouet, "Antioxidant strategies in the treatment of stroke," Free Radical Biology and Medicine, vol. 39, no. 4, pp. 429-443, 2005.

[16] P. A. Kocaturk, M. C. Akbostanci, C. Isikay, et al., "Antioxidant status in cerebrovascular accident," Biological Trace Element Research, vol. 80, no. 2, pp. 115-124, 2001.

[17] P. Bertrand, M. Starck, B. Herbeth, M. Vincent-Viry, F. Schiele, and G. Siest, "Serum total antioxidant status is higher in postmenopausal women and after estrogen replacement therapy," Clinical Chemistry and Laboratory Medicine, vol. 40, no. 8, pp. 850-852, 2002. 
[18] B. Moosmann and C. Behl, "The antioxidant neuroprotective effects of estrogens and phenolic compounds are independent from their estrogenic properties," Proceedings of the National Academy of Sciences of the United States of America, vol. 96, no. 16, pp. 8867-8872, 1999.

[19] D. Amantea, R. Russo, G. Bagetta, and M. T. Corasaniti, "From clinical evidence to molecular mechanisms underlying neuroprotection afforded by estrogens," Pharmacological Research, vol. 52, no. 2, pp. 119-132, 2005. 

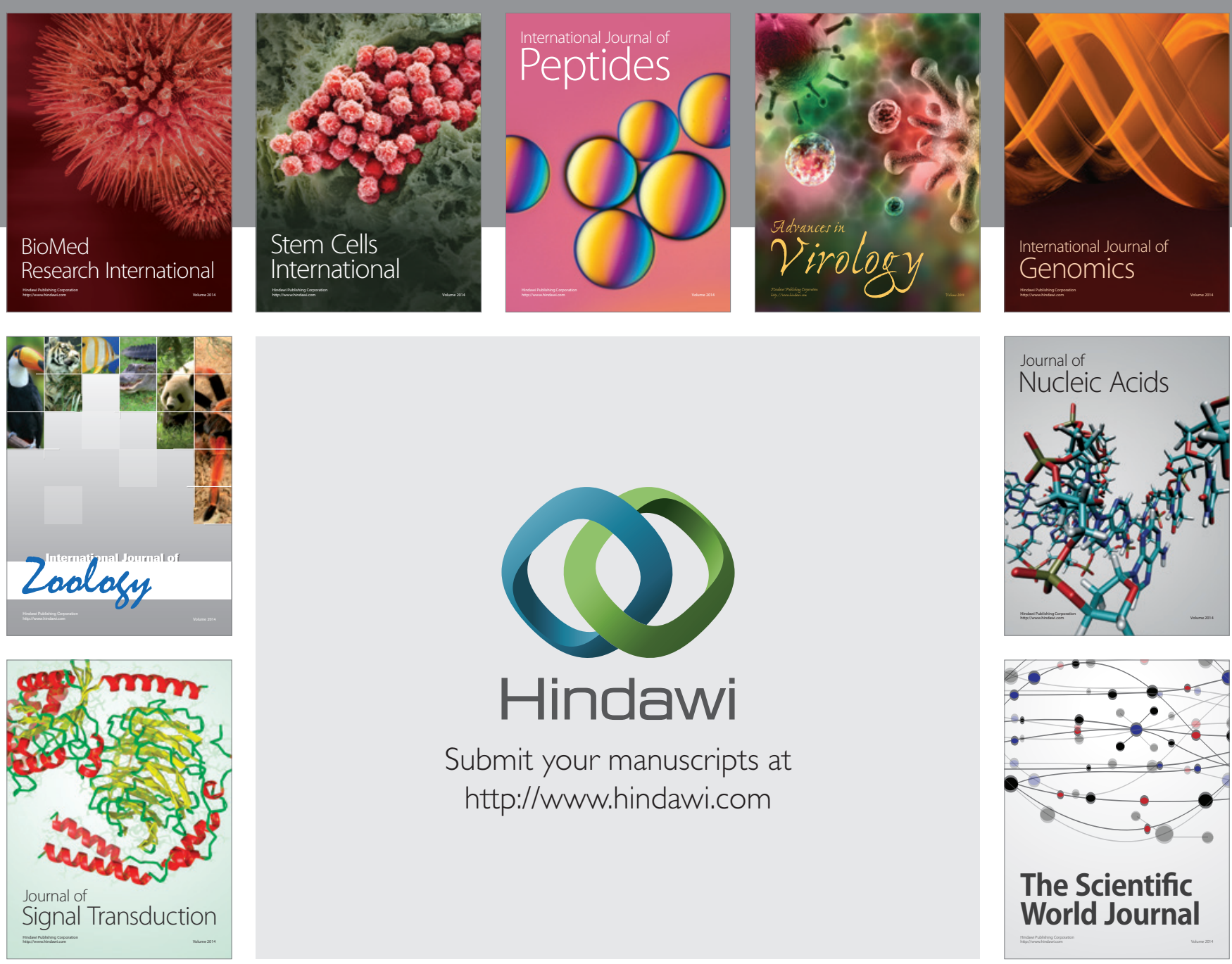

Submit your manuscripts at

http://www.hindawi.com
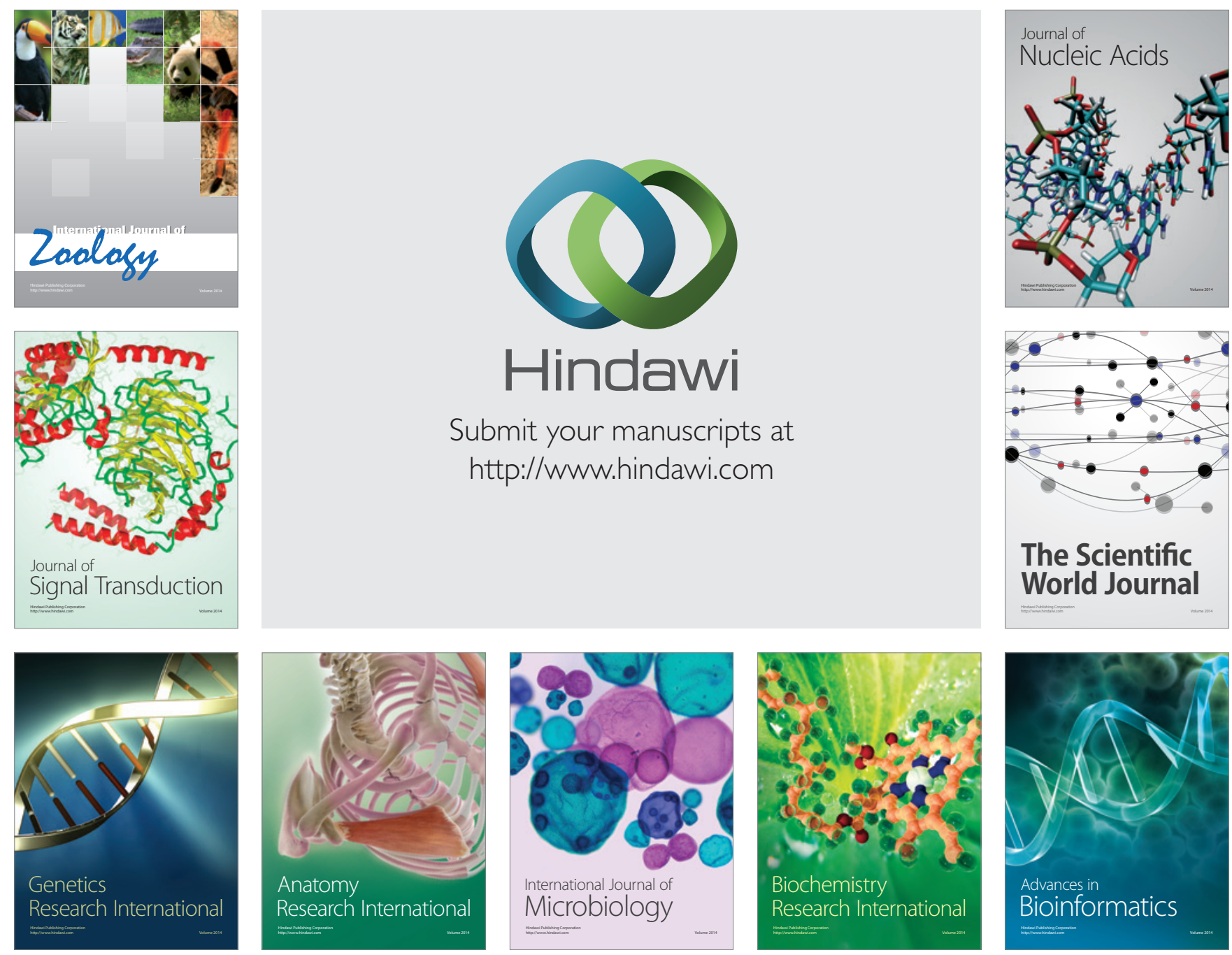

The Scientific World Journal
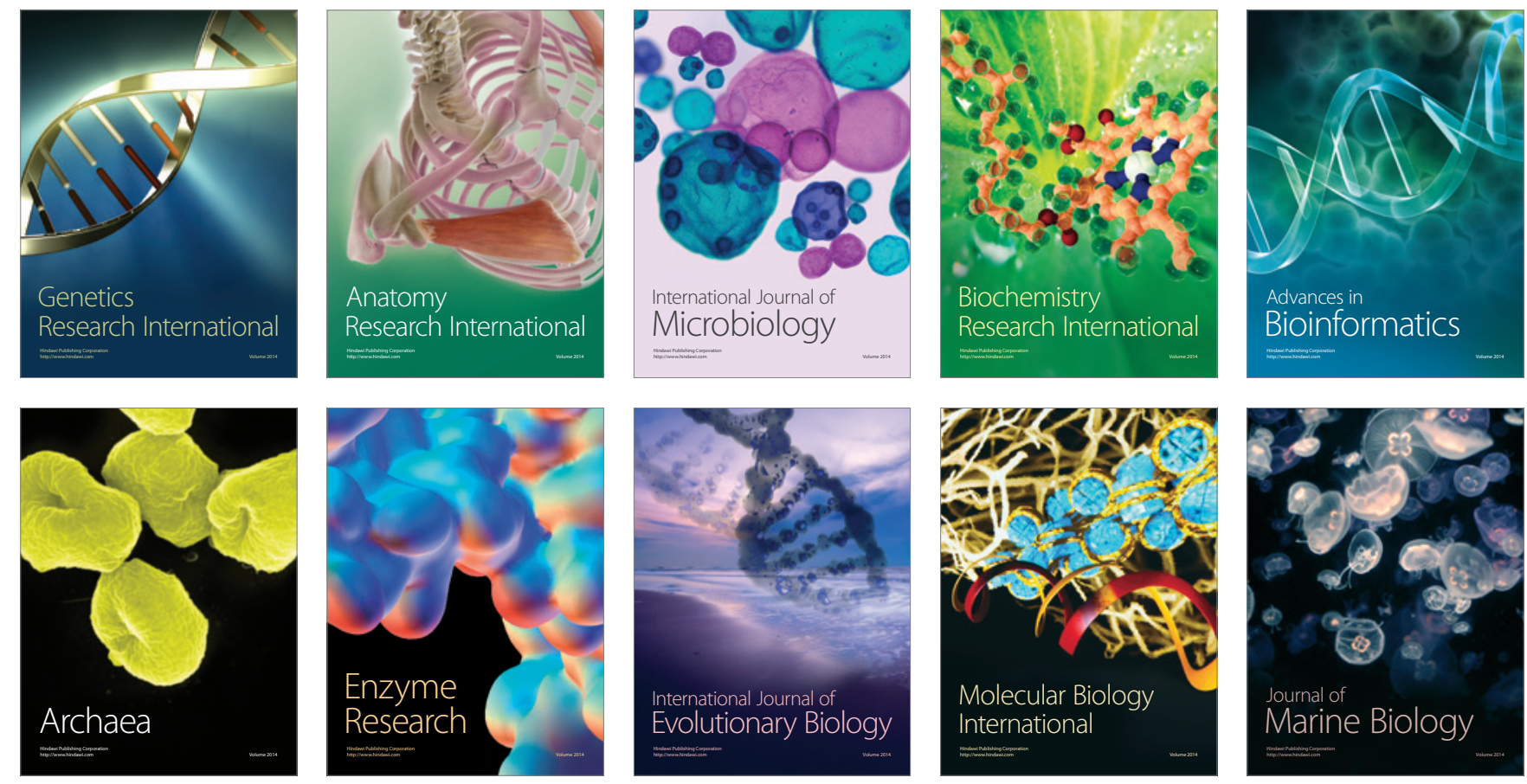